MILITARY TECHNICAL COLLEGE CAIRO - EGYPT

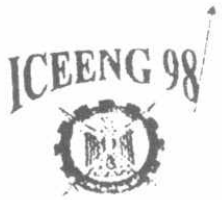

1st INTERNATIONAL CONF. ON

ELECTRICAL ENGINEERING

\title{
Closed Form Solution Of A Class Of Nonlinear Circuits Using Normal Forms
}

\author{
Abdel-Karim Aboul-Hassan \\ Faculty of Engineering, Alexandria University, Alexandria, Egypt \\ Abdel-Monem Abdel-Bary Nasser \\ Air Defense college, Alexandria, Egypt \\ Abdel-Azeem Mohamed \\ Air Defense college, Alexandria, Egypt
}

\begin{abstract}
This paper presents a method for obtaining closed form expressions for a class of nonlinear circuits of autonomons and nonatomomons types. The method deperds on the theory of normal forms. It overcomes the drawbacks confronted by other methods such as harmonic balance, volterra series and direct integration. This method also is suitable for the investigation of periodic, quasiperiodic and chaotic behaviors.
\end{abstract}

\section{Introduction:}

Many circuit applications utilize nonlinear elements such as nonlinear resistors, nonlinear inductors and nonlinear capacitors of voltage or current controlled types. Mathematical models of circuits which are differential systems are derived and analyzed by many authors [1],[2],[3]. These differential systems may be linear or nonlinear. The classical equations of Lienard and Vanderpol are studied from the purely mathematical point of view and from the applied one. It has been found in[4] that some properties of these equations are not possessed by linear flows. Moreover in [5] Lienard's equations are linked to the formidable Hilbert's 16th problems where one form of Lienard's equations has polynomial vector fields

The problem of reducing a system of ordinary differential equations to its normal form was formulated by Poincare [6]. Results of general character were obtained later and to be found in the references [7], [8], [9]. The present work uses the method of normal forms for the investigation of some circuits aiming at singling out general classes amenable to such methods. In studying dynamical systems from qualitative point of view one usually looks for periodic, quasiperiodic or chaotic behavior. Chaos may be considered as a kind of behavior that follows period doubling bifurcation, period adding, intermittency or quasiperidicity.

The ever increasing applications of chaotic systems which include stabilization and control, multimode laser generation, chemical reactions control, heart arhythmias 
prediction, biological neural networks and encryptioninspired the study of chaotic behavior in circuits and systems. One of the efficient tools in the investigation of chaos is the method of normal forms [9-13].

\section{Normal Forms :}

Consider the differential system:

where,

$$
\dot{X}=A X+U_{n}(x)+U_{i}(t)
$$

$A$ is a (nxn ) matrix, $U_{n}(x)$ is the vector of the nonlinear functions and $U_{i}(t)$ is the source vector. $U_{n}(x)$ satisfies some regularity conditions [1]. There exists a vast literature on the problems of reducing equation (1) to its normal form including theorems such as classical normalization theorem, Poincare linearlization theorem, Hartman - Grobman theorem ,Ushiki's method etc... [3], [4], [11].

\subsection{Normal forms of linear systems:}

The normal form of a liner system depends mainly on the concept of Linear Algebra This method transforms an A (nxn ) matrix to a simple from ( diagonal, Jordan , ...). This form facilitates the calculation of $\exp (\mathrm{tA})$ that results in the solution of a system of differential equations. If $\mathrm{A}$ is in normal form the limit sum of exp(tA) is convergent otherwise it is divergent, and hence the solution of the system of differential equations becomes not easy [14]

\subsection{Normal form of nonlinear systems:}

The nonlinear system may be linearized, but not all nonlinear systems can be linearized, Pioncare and Hartman - Grobman give some rules for testing the nonlinear system for linearization. These rules depend on the shape of the Jacobi matrix and its eigenvalues. If the system is not linearizable we have to use other concepts following Takens, Ushiki and Chua. [7 ], [ 11$],[12]$.

In what follows examples for lumped and distributed circuits in different orders are given and treated using normal form methods to illustrate the efficiency of these methods .

\section{Examples}

\section{Example 3.1}

The circuit shown in Fig. (1) is modeled by the following differential system

$$
\mathrm{X}=\mathrm{AX}+\mathrm{U}_{\mathrm{i}}(\mathrm{t})
$$

where, 


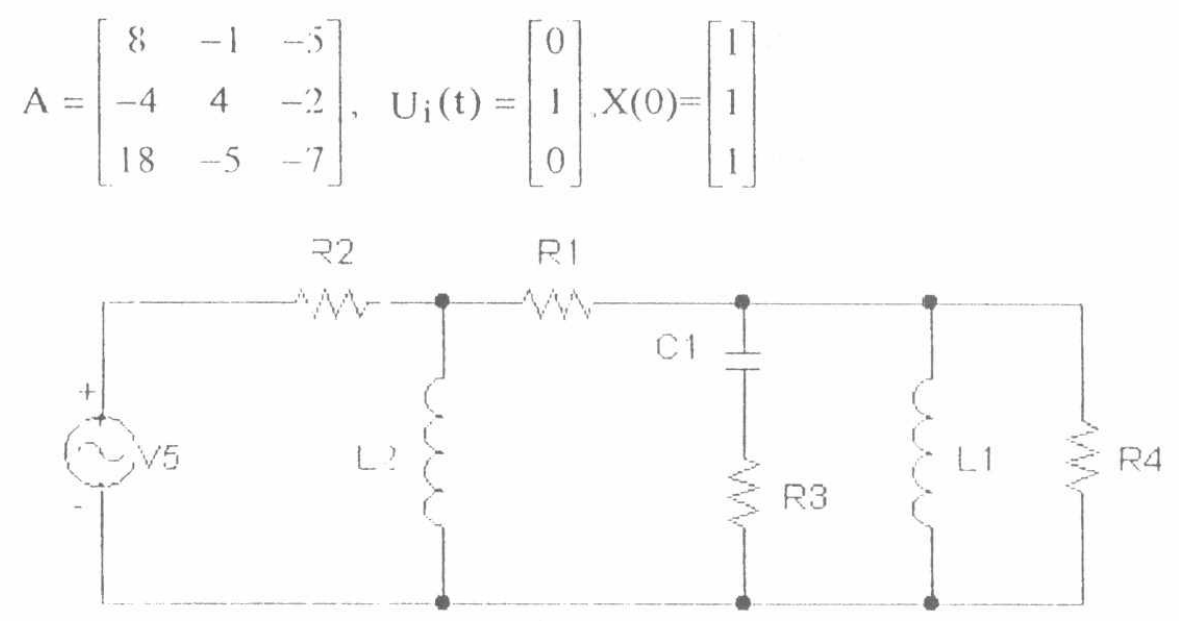

Fig. $13^{\mathrm{rd}}$-order RLC circuit

First wa substitute $X()=P Y(t)$,

where $P$ is a nonsingular matrix then we get the following reduced(Normal form) :

$$
\mathrm{Y}=\mathrm{BY}+\mathrm{j}(\mathrm{t}
$$

where,

$$
\left.B=\left[\begin{array}{ccc}
0 & 0 & 20 \\
1 & 0 & -24 \\
0 & 1 & 5
\end{array}\right], \quad \mathrm{j} t\right)=\left[\begin{array}{c}
\frac{-35}{68} \\
\frac{19}{680} \\
\frac{-9}{680}
\end{array}\right]
$$

Thus the solution of equation (3) is

$$
Y(t)=Y(0) e^{B t}+e^{B t} \int_{0}^{t} j(t) e^{-B t} d t
$$

Hence $X(t)=P Y(t)$ w hich takes the form

$$
\left[\begin{array}{c}
-\frac{9}{10}+\frac{9}{10} e^{(2 x)} \cos (4 x)-\frac{1}{5} \mathrm{e}^{(2 x)} \sin (4 x)+\mathrm{e}^{x} \\
-\frac{17}{10}+\frac{7}{10} \mathrm{e}^{(2 x)} \cos (4 x)-\frac{11}{10} \mathrm{e}^{(2 x)} \sin (4 x)+2 \mathrm{e}^{x} \\
-\frac{11}{10}+\frac{11}{10} \mathrm{e}^{(2 x)} \cos (4 x)+\frac{7}{10} \mathrm{e}^{(2 x)} \sin (4 x)+\mathrm{e}^{x}
\end{array}\right]
$$

This example has been solved in [14] by another method and the solution is in agreem ent with their s esults. The normal form has the advantage of being a direct method

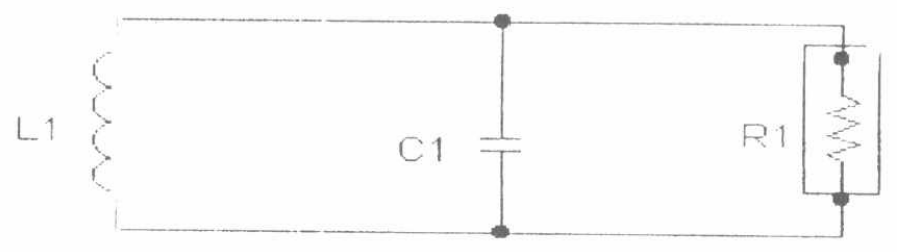

Fig. 2. RLC circuit with one resistor characteristic(nonlinear circuit) 


\section{Example 3.2}

The circuit shown in Fig.(2) is modeled by the following differential system:

$$
\begin{aligned}
& x^{\cdot}=-y-x^{3}, \\
& y=x
\end{aligned}
$$

Poincare' linearlization theorem is not feasible in this case as well as Hartman - Grobman theorem. Thus we apply Ushiki' method [7 ], [12]. This method depends mainly on computing Lie brackets and the $\mathrm{k}$-jet. To obtain the kth-order normal form we have to solve the following equation for $g_{k}$.

Let $\chi_{0}$ be the vector space of all smooth vector fields (i.e., $C^{\infty}$ ),

$\chi_{0}^{k} \quad$ be the vector space of all $K$-jet of the vector fields in $\chi_{0}$ at 0 (obtained by truncating all terms of degree greater than $\mathrm{k}$ ),

$\mathrm{H}_{h} \quad$ is the subspace consisting of all vector fields described by a homogeneous polynomial of degree $\mathrm{k}, \mathrm{H}_{\mathrm{k}} \subset \chi_{0}^{\mathrm{k}}$.

$B_{k} \quad$ is the subspace of $H_{k}$ consisting of the image of the linear mape, $B_{k} \subset H_{k}$,

$\mathrm{G}_{\mathrm{k}}$ is the complementary subspace to $B_{k}$,

$$
\frac{d}{d t} g_{k}(t)=-\pi_{k}\left(\left[Y^{k-1}, V^{k-1}+g_{k}(t)\right]_{k}\right)
$$

with $\left[Y^{k-1}, V^{k-1}\right]^{k-1}=0$, where, $\left[Y^{k-1}, V^{k-1}\right]^{k-1}$ is the k-jets of $\left[Y^{k-1}, V^{k-1}\right]$ at 0 ,

$\pi_{k} \quad$ is the natural projection of $H_{k}$ along $B_{k}$ by $\pi_{k}: H_{k} \rightarrow G_{k}$, $V^{k} \in X_{0}{ }^{k}, Y_{k} \in H H_{h} .\left[Y^{k-1}, V^{k-1}\right]$ is the lie bracket of $Y^{k-1}$ and $V^{k-1}$,

The solution of equation (6) leads to kth-order normal form. The successive normal forms of the system given by equation (5) are:

(i) $1^{\text {st }}$ order normal form

form(equivalent to linear part).

(ii) $2^{\text {nd }}$ order normal form

The system have jacobi mat rix of the form $\left[\begin{array}{cc}0 & -1 \\ 1 & 0\end{array}\right]$. The second order component in the normal form is completely eliminated.

(iii) $3^{\text {rd }}$ order normal form

(a) Non-degsenerate case

$$
\pm\left(x^{2}+y^{2}\right)(x \delta \delta x+y \delta \delta y)+\left(1+\beta\left(x^{2}+y^{2}\right)\right)(-y \delta \delta x+x \delta \delta y)
$$

where,

$\beta$ is the arbitrary constant from the integration equation (6)

(b) Deg,enerate case $(\beta=0)$

$$
\left(1 \pm\left(\mathrm{x}^{2}+\mathrm{y}^{2}\right)\right)(-\mathrm{y} \delta \delta \mathrm{x}+\mathrm{x} \delta \delta \mathrm{y})
$$


Proccedings of the $1^{\text {st }}$ ICEENG Conf. 24-26 March 1998

following the same rocedure airove we can find the $k^{\text {th }}$ order normal form of above syster

(iv) $\mathrm{k}^{\text {th }}$ order normal form

The non-idege nerate $k$-th order normal form of the vector tield on $\mathrm{R}^{2}$ is given by

$$
\left( \pm r^{2}+\alpha r^{+}\right) r \delta \delta r+\left(1+\beta r^{2}\right) \delta \delta \theta
$$

for any $k \geq 5$, where $(r, \theta)$ are the polar coordinates

The state - trajectory associated with the system is shown in Fig.(3) where a limit cycle solution appears at different initial condition for degenerate and non degenerate cases thus one can study different types of behavior without resorting the pattern of the original
eigenvialues

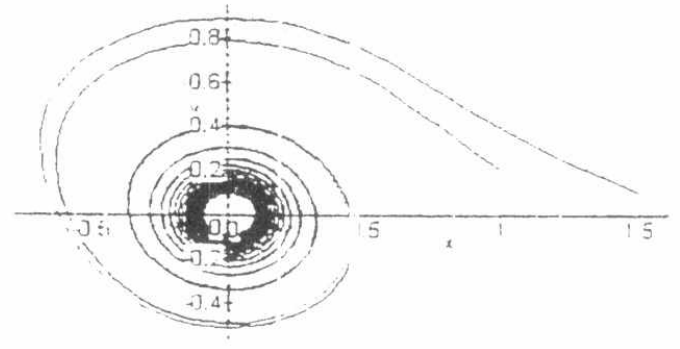

(a)

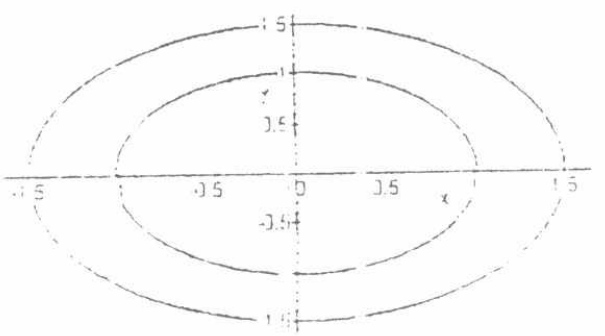

(c)

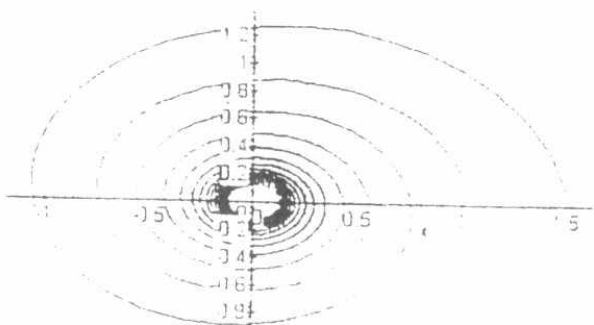

(b)

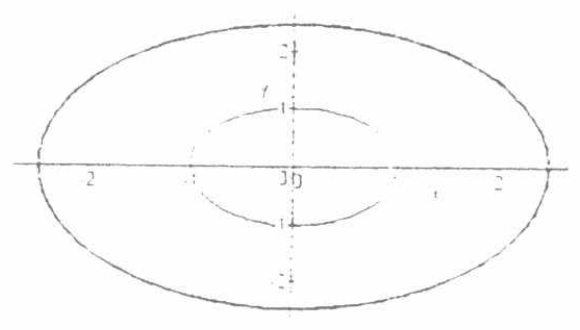

(d)

Fig. $\therefore$ (a! $\beta=2.1$. (b) $\beta=9.3$. (c) $\beta=0, x(0)=1.2$. (d) $\beta=0, x(0)=2.6$ 


\section{Example 3.3}

The circuit shown in Fig.(4) is modeled by the following differential system:

$$
\begin{aligned}
& x^{\cdot}=y-x+x^{3}, \\
& y^{\cdot}=y
\end{aligned}
$$

Following the same procedure in example 3.2 we can solve system in equation (7) and get the following results

(i) $1^{\text {st }}$ order normal form

As the system is not linearizable we can not get the first order normal form(equivalently to linear part).

(ii) $2^{\text {"ul }}$ order normal form

The system have jacobi matrix of the form $\left[\begin{array}{cc}-1 & 1 \\ 0 & 1\end{array}\right]$. The second order component in the normal form is completely eliminated.

(iii) $3^{\text {rd }}$ order normal form

(a) Non-degenerate case

$$
((y-x) \delta \delta x+y \delta \delta y)+\left( \pm x^{2} y+\beta x^{3}\right)(\delta \delta y)
$$

(b) Degenerate case $(\beta=0)$

$$
((y-x) \delta \delta x+y \delta \delta y)+\left( \pm x^{2} y\right)(\delta \delta y)
$$

Note that : this system have only $3^{\text {rd }}$ order normal form and all $k^{\text {th }}$ order component in $k^{\text {th }}$ order normal form for $\mathrm{k}>3$ is conpletely efiminated.

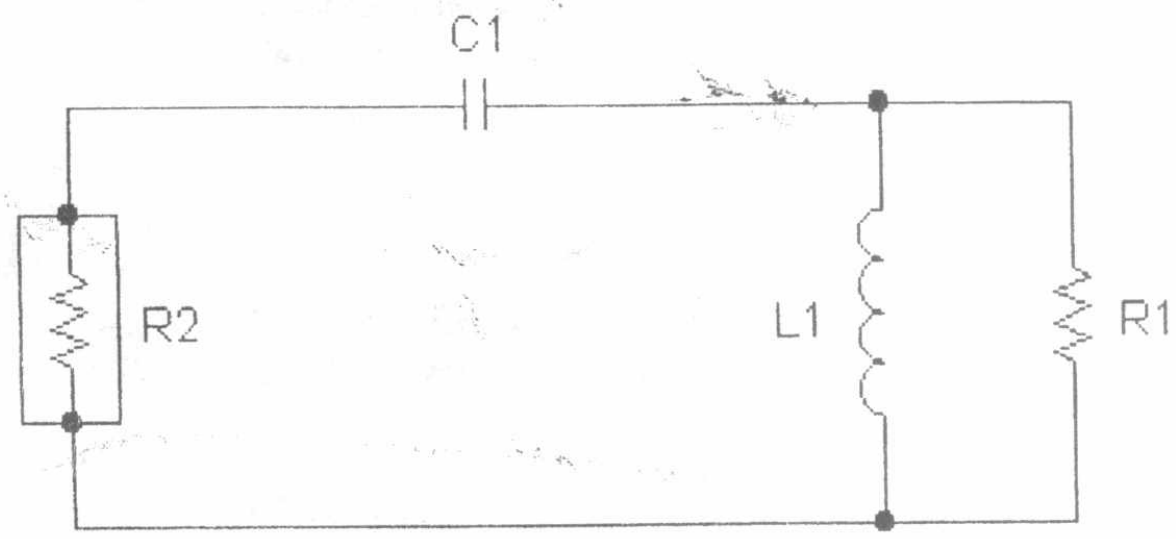

Fig. 4. RLC circuit with one resistor characteristic(nonlinear circuit)

The state - trajectory associated with the system is shown in Fig.(5) where a limit cycle solution appears at different initial condition for degenerate and non degenerate cases thus one can study different types of behavior without resorting the pattern of the original eigenvalues. 
Proceedings of the $1^{\text {st }}$ ICEENG Conf. 24-26 March 1998

(a)

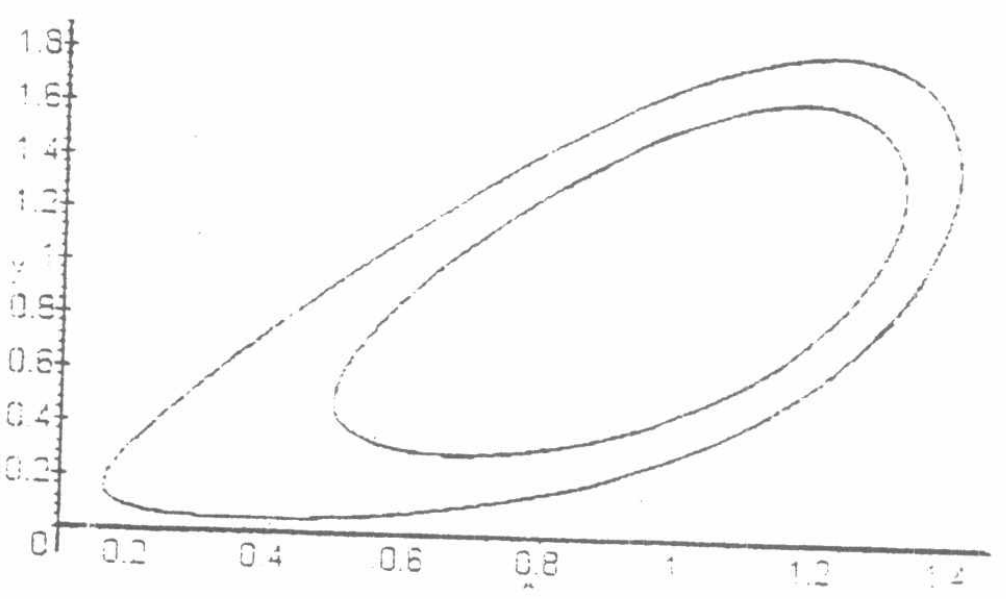

(b)

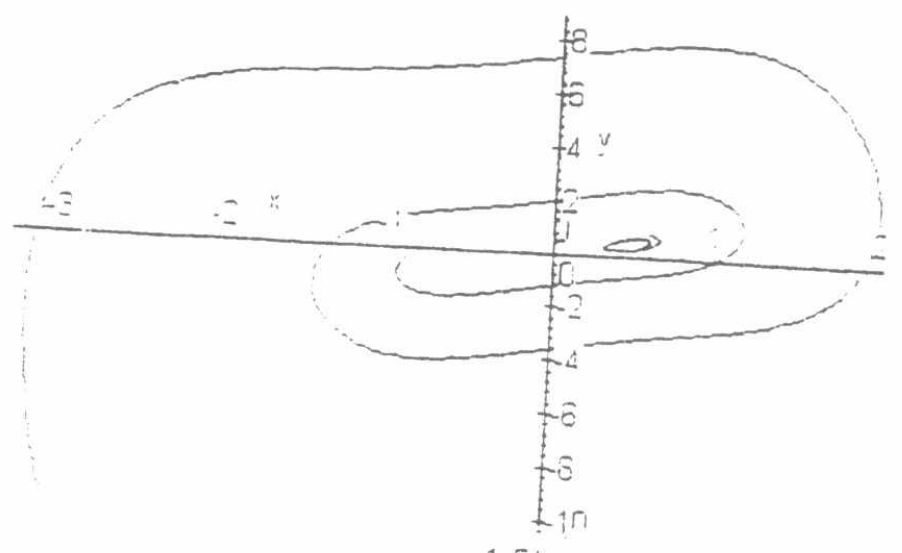

(c)

(d)

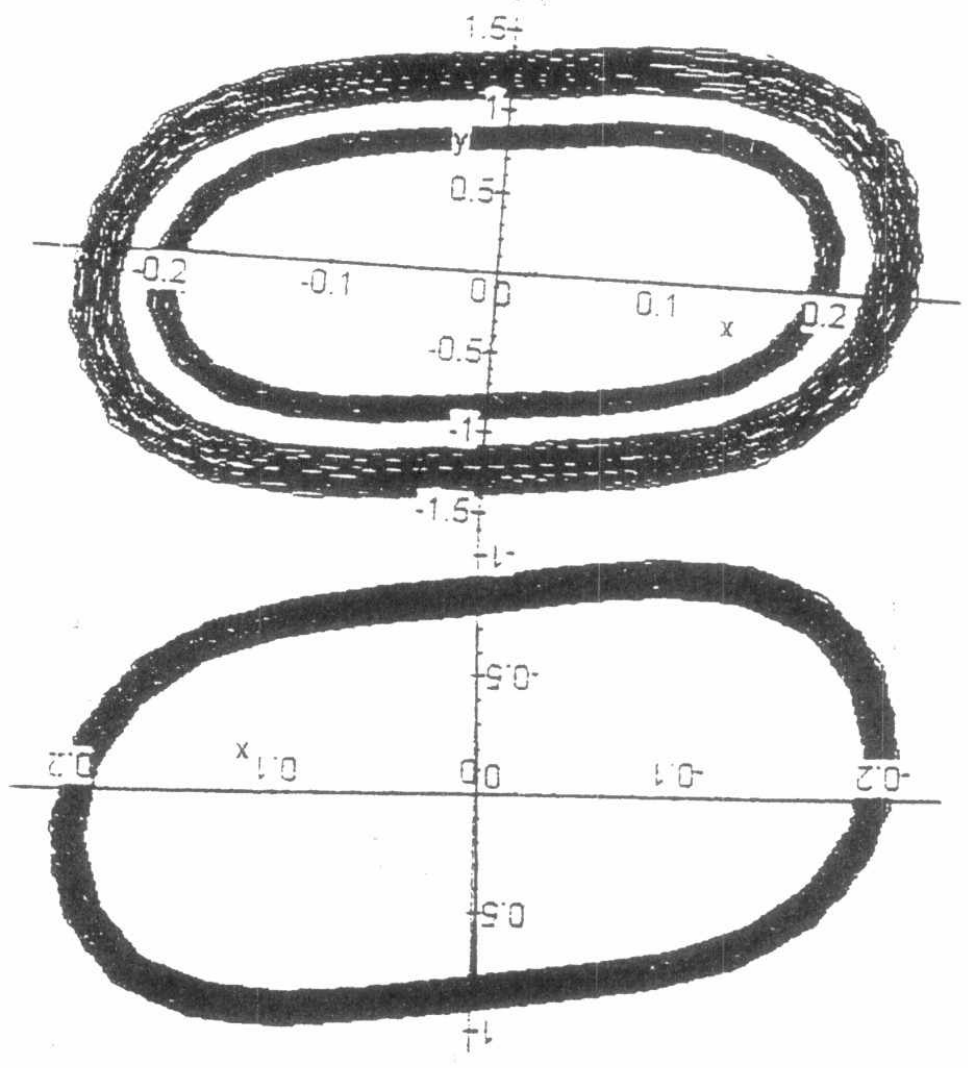

Fig.5. (a) $\beta=0$ (b) $\beta=-3.3$ (c) $\beta=-822.3$ (d) $\beta=-1062.4$ 


\section{Example 3.4}

The circuit shown in Fig.(6) (nonlinear distributed oscillator is modeled by the following system of difference equations[15]:

$$
\left[\begin{array}{c}
V_{\overline{1 i}}^{-}(t+T) \\
V_{10}^{-}(t+T)
\end{array}\right]=\left[\begin{array}{cc}
0 & 1 \\
\rho_{L} & 0
\end{array}\right]\left[\begin{array}{c}
V_{\overline{l i}}^{-}(t) \\
V_{\overline{10}}^{-}(t)
\end{array}\right]+\left[\begin{array}{c}
0 \\
-Z_{0} * U_{n}\left(V_{1 i}(t)\right)
\end{array}\right]
$$

where

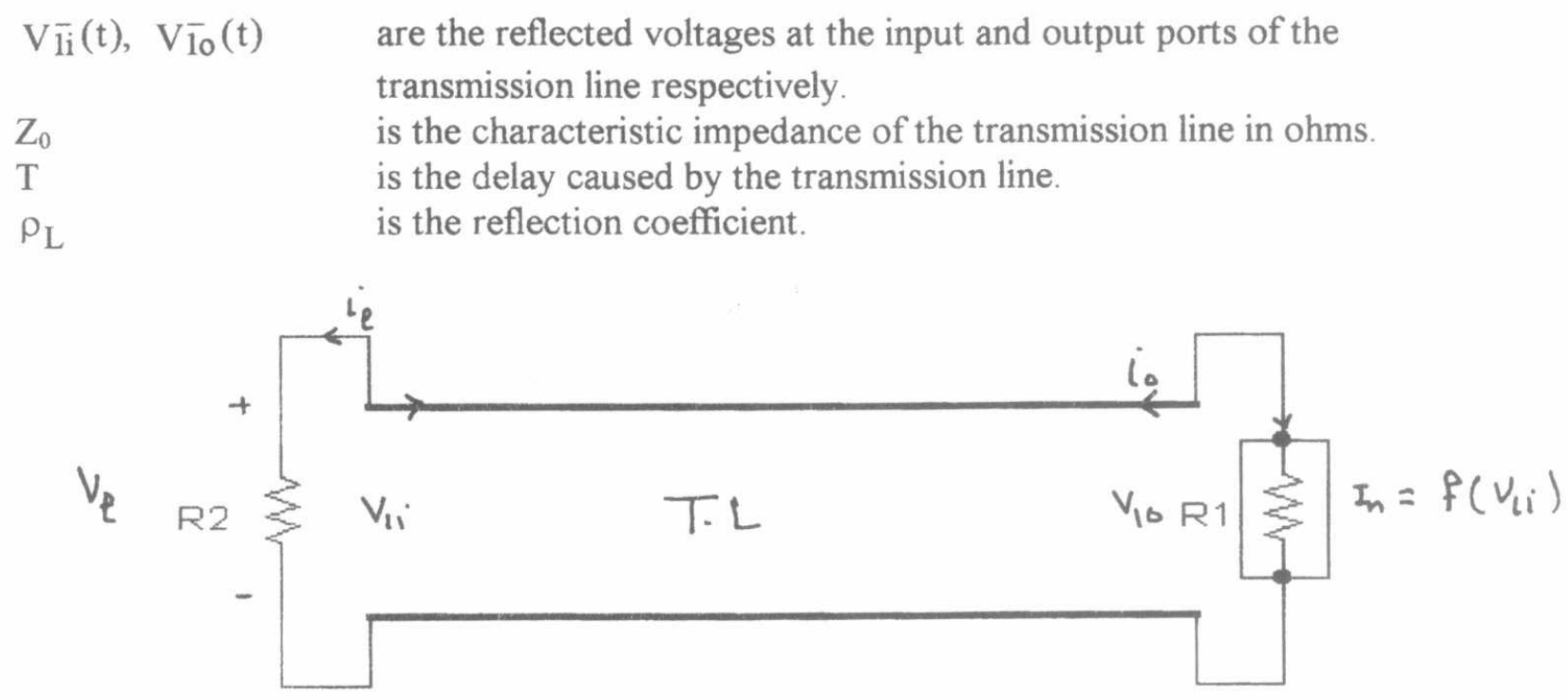

Fig. 6. Transmission Line(nonlinear distributed oscillator)

The difficulty in solving such system lies in the fact that the argument of the nonlinear function $\mathrm{U}_{\mathrm{n}}$ is given as a total voltage $\mathrm{V}_{1 i}(\mathrm{t})$.

$$
\begin{aligned}
& \mathrm{U}_{\mathrm{n}}\left(\mathrm{V}_{1 \mathrm{i}}(\mathrm{t})\right)=-\mathrm{V}_{1 \mathrm{i}}(\mathrm{t})+\mathrm{V}_{1 \mathrm{i}}{ }^{2}(\mathrm{t}) \\
& \mathrm{V}_{\mathrm{li}}(\mathrm{t})=\mathrm{V}_{\overline{\mathrm{ii}}}(\mathrm{t})+\mathrm{V}_{\mathrm{li}}^{+}(\mathrm{t}) \\
& =\mathrm{V}_{\mathrm{1i}}(\mathrm{t})+\mathrm{V}_{\overline{10}}(\mathrm{t}+\mathrm{T})
\end{aligned}
$$

Substituting into equation (8) we get,

$$
\mathrm{U}_{\mathrm{n}}\left(\mathrm{V}_{\mathrm{li}}(\mathrm{t})\right)=-\mathrm{V}_{\overline{\mathrm{i}}}(\mathrm{t})-\mathrm{V}_{\overline{10}}(\mathrm{t}+\mathrm{T})+\left(\mathrm{V}_{\mathrm{li}}(\mathrm{t})\right)^{2}+2 \mathrm{~V}_{\overline{\mathrm{i}}}(\mathrm{t}) \mathrm{V}_{\overline{10}}(\mathrm{t}+\mathrm{T})+\left(\mathrm{V}_{\overline{10}}(\mathrm{t}+\mathrm{T})\right)^{2}
$$

Because the system in equation (6) is in the normal form, one is able to rearrange the system by substituting $\quad \mathrm{V} \overline{1}_{\mathrm{o}}(\mathrm{t}+\mathrm{T})=\rho_{\mathrm{L}} \mathrm{V}_{\overline{1}} \mathrm{i}(\mathrm{t}) \quad$ in equation (8), then the new system will take the following form.

$$
\left[\begin{array}{c}
\mathrm{V}_{\overline{\mathrm{li}}}(\mathrm{t}+\mathrm{T}) \\
\mathrm{V}_{\overline{\mathrm{lo}}}(\mathrm{t}+\mathrm{T})
\end{array}\right]=\left[\begin{array}{cc}
0 & 1 \\
\rho_{\mathrm{L}} & 0
\end{array}\right]\left[\begin{array}{c}
\mathrm{V}_{\overline{\mathrm{li}}}(\mathrm{t}) \\
\mathrm{V}_{\overline{\mathrm{lo}}}(\mathrm{t})
\end{array}\right]+\left[\begin{array}{c}
0 \\
\mathrm{Z}_{0} *\left(1+\rho_{\mathrm{L}}\right) \mathrm{V}_{\overline{\mathrm{li}}}(\mathrm{t})\left(1-\left(1+\rho_{\mathrm{L}}\right) \mathrm{V}_{\overline{\mathrm{li}}}(\mathrm{t})\right)
\end{array}\right]
$$



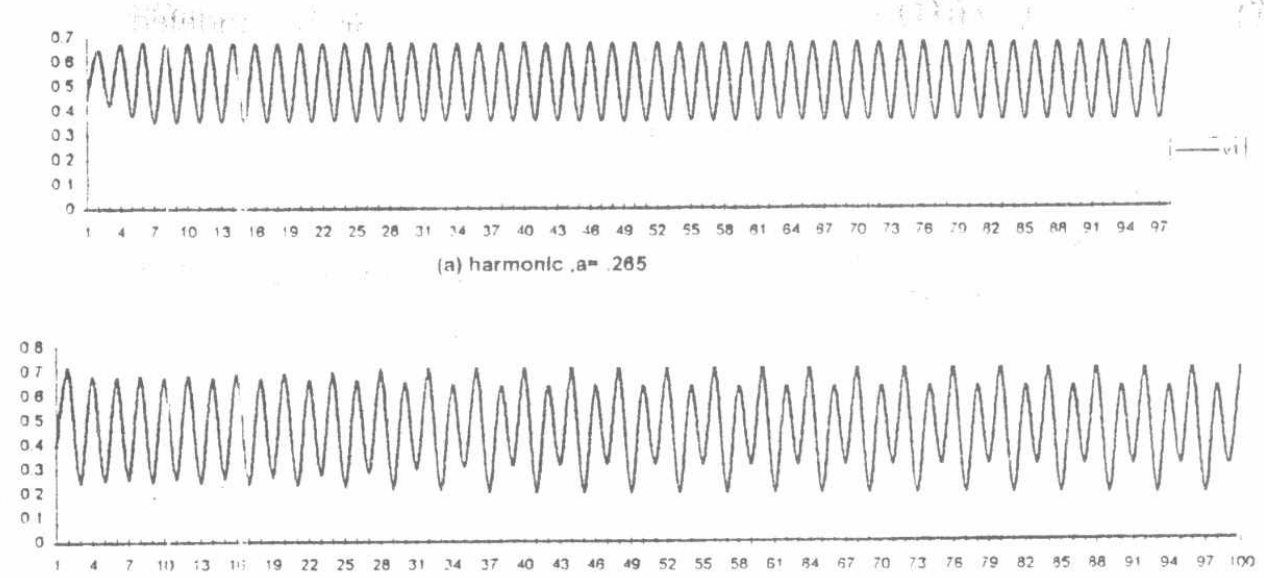

(b) period doubling , $a=345$

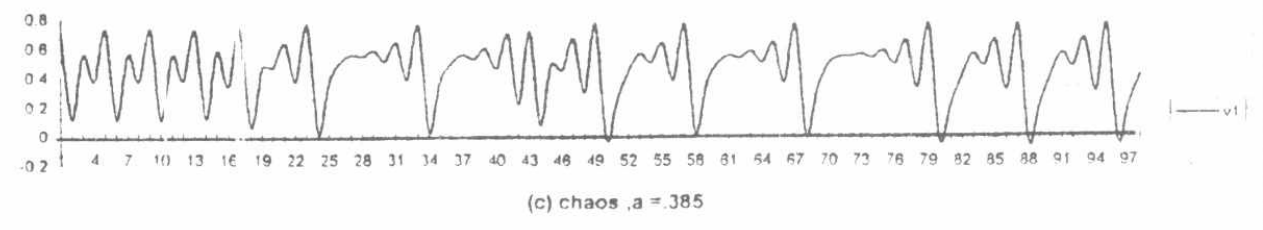

Fig. 7.(a) harmonic.(b) period doubling.(c)Chaos

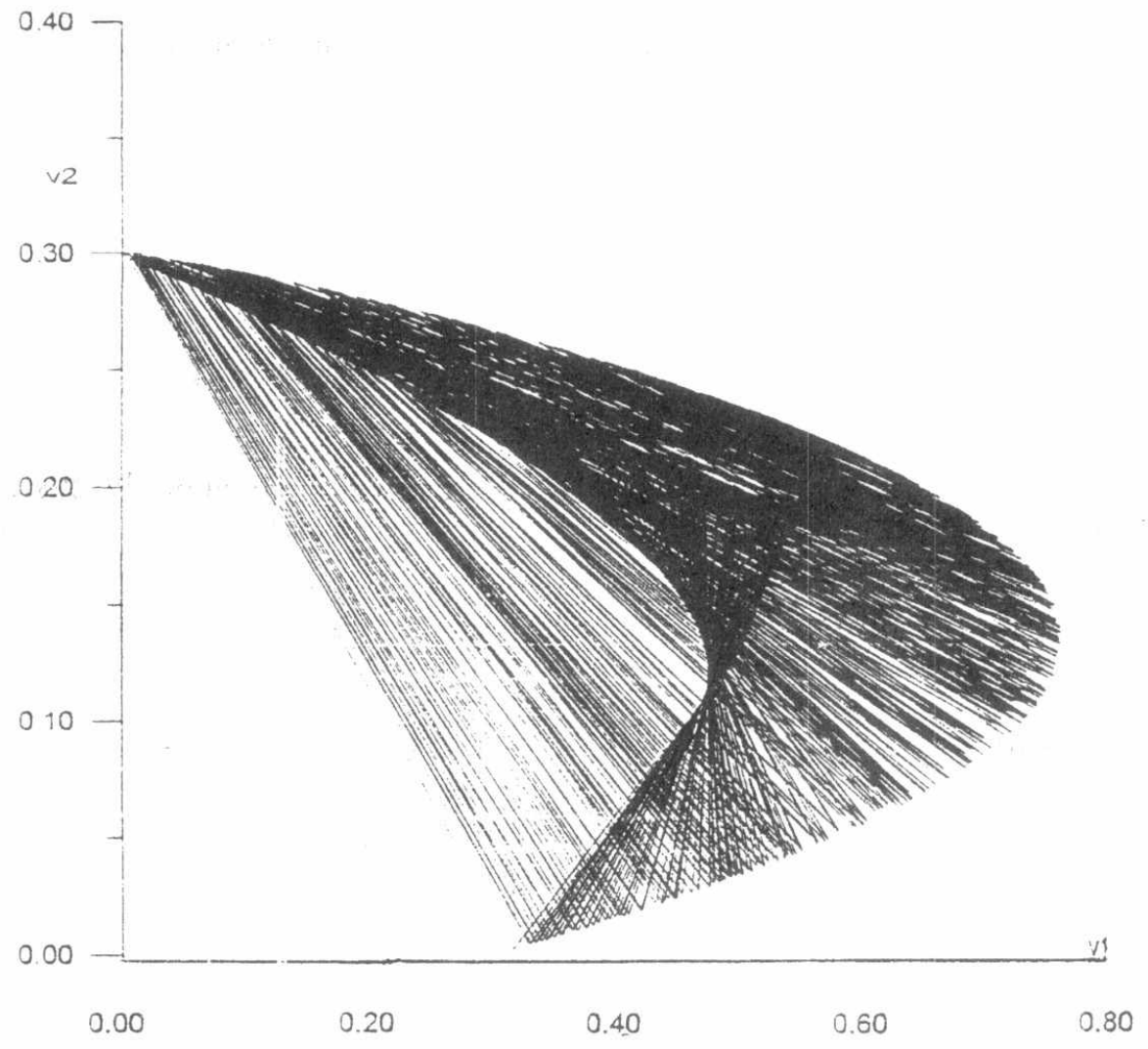

Fig. 8. Chaos . $\rho_{\mathrm{L}}=0.385$ 
The above system can be easily solved by a simple iterative method. Different types of behavior can be predicated as shown in Fig.( 7) In this figure harmonic, period doubling, and chaotic time domain solution are given with the corresponding values of the bifurcation

parameter $\rho_{\mathrm{L}}$ (the reflection coefficient). The attractor of the circuit in the chaotic region is shown in Fig.(8). This again shows the simplicity gained by the normal form solution of the nonlinear system .

\section{Conclusion:}

Numerical methods are used extensively in handling electrical circuit analysis problems with advantages and disadvantages. There is no general numerical algorithm to handle different types of nonlinearity (weak or strong nonlinearity) as well as high dimensionality. Closed form solutions are then needed to overcome the disadvantages of the numerical methods. The present work furnishes the efficiency of using the normal form methods. Different types of behaviors are detected such as periodicity, quasiperidicity and chaos. This is a first step to single out a class of circuits amenable to normal form methods.

\section{References:}

[1]S.Smale," on the mathematical foundations of electrical circuit theory", J. Differential Geom

210. 1972

[2]L.O.Chua, C.A.Desoer, and E.S.Kuh, "Linear and nonlinear circuits".NewYork: McGraw-Hill,1987.

[3]J.Guckenheimer and P.J.Holmes, "Nonlinear oscillations, Dynamical systems, and Bifurcations of vector fields". NewYork: Springer-verlag, 2nd printing, 1983.

[4]J.Della Dora, L.Stolovitch, "Normal forms of differential systems". London Mathematical Society, Lecture Note Series 193, CAMBRIDGE UNIVERISITY PRESS

[5] A.Liens, W.D.Melo and C.C.Pugh, “ on Lineard's equation”, Lecture In Mathematical Springer.597, 1977

[6] Poincare',H. "Les methodes Nouvells de la mecanique celeste", 3 vols. Gauthier villars : paris, 1899.

[7] F. Takens, "Normal form for certain singularities of vector fields", nn. Inst. Fourier, vol.23, pp. 163-195,

[8]S.Ushi, "Nothods in ", in computing methoution science and engineering, VI, R. Glowinski and J. L. Lions, Eds.Amesterdam, The netherlands: North Holand, 1984.

[9]K.R.Meyer, "perturbution analysis of nonlinear systems". London Mathematical Society, Lecture Note Series 193, cambridge univerisity press, 1995.

[10] K.Aihira and R.Katayama, "Chaos engineering in Japan,".Communications of the ACM, vol.38.,1995.

[11] S.Ushiki, "Normal form for singularities of vector fields"," Japan J.Appl.Math., Vol.pp. 1-37, 1984

[12]L.O.Chua and H.Kokubu, "Normal forms for nonlinear vector fields-part 1 : Theory and algorithm", "IEEE Circuits syst.,vol.35,pp 863-880, 1987.

[13]L.O.Chua and H.Kokubu, "Normal forms for nonlinear vector fields-part 2 : Applications", "IEEE ciruits and syst.,vol.36,pp 863-880, 1989.

[14]Mojatabaa Chinichiaan and Charles T. Fulton, "Closed -Form solution of linear Time -Invariant differential system".IEEE Circuits and Devices, vol.2 , No. 3, 1986"

[15]M. I. Sobhy, E. A. Hosny, and A. A. A. Nasser, "systematic analysis procdure for chaotic curcuits transmission lines", proc. of the $10^{\text {th }}$ European cof. on circuit theory and and desigen , ECCTD-91, vol.III,

pp. 1253-1262. the Techincal univerisity of Deumark, Copenhagen, sep . 1991. 
